\title{
Mutation spectrum analysis of 29 causative genes in 43 Chinese patients with congenital hypothyroidism
}

\author{
HUIJUAN WANG $^{1 *}$, XIAOHONG KONG ${ }^{1}$, YANRUI PEI ${ }^{1}$, XUEMEI CUI ${ }^{2}$, YIJIE ZHU ${ }^{3}$, \\ ZIXUAN HE ${ }^{4}$, YANXIA WANG ${ }^{1}$, LIRONG ZHANG $^{1}$, LIXIA ZHUO $^{1}$, CHAO CHEN $^{1}$ and XIAOLI YAN ${ }^{5}$ \\ ${ }^{1}$ The National Engineering Research Center for Miniaturized Detection Systems, College of Life Science, \\ Northwest University, Xi'an, Shaanxi 710069; ${ }^{2}$ The Tianyou Children's Hospital of Xi'an, Xi'an, Shaanxi 710061; \\ ${ }^{3}$ The Chang An Hospital, Xi'an, Shaanxi 710016; ${ }^{4}$ Beijing Shijitan Hospital, Beijing 100080; \\ ${ }^{5}$ Endocrine Department, Xi'an Children's Hospital, Xi'an, Shaanxi 710003, P.R. China
}

Received May 28, 2019; Accepted February 13, 2020

DOI: $10.3892 / \mathrm{mmr} .2020 .11078$

\begin{abstract}
Congenital hypothyroidism $(\mathrm{CH})$ is the most common neonatal endocrine disorder with a genetic origin. The purpose of the present study was to analyze the mutation spectrum of $\mathrm{CH}$ patients in China. A targeted next-generation sequencing panel covering all exons of $29 \mathrm{CH}$-related causative genes was used in 43 Han Chinese patients with $\mathrm{CH}$ [11 dysgenesis and 32 glands in situ (GIS)]. The functional impact and pathogenicity of detected variants were analyzed using a comprehensive bioinformatics approach and co-segregation studies. A total of 47 rare non-polymorphic variants in 9 target genes associated with thyroid hormone synthesis (DUOX2, DUOXA2, TPO, TG, SLC26A4 and SLC5A5), thyroid stimulating hormone resistance (TSHR) and central hypothyroidism (PROP1 and TRHR) were identified in 31 patients $(31 / 43$, $72 \%)$. Of these variants, 8 were novel, including 3 in DUOX2, 2 in TPO, 3 in TSHR and 1 in SLC5A5. Variants were mostly affected by $D U O X 2, T G, T P O$ and TSHR. Approximately $44 \%$ of the patients $(19 / 43)$ carried DUOX2 variants. The mutation detection rates in patients with GIS were higher compared with patients with dysgenesis [25/32 (78\%) vs. 6/11 (54\%)]. Oligogenic mutations were detected in $25.6 \%$ of the total cases and $35 \%$ of the mutated cases. Genetic basis was ascertained in 13 patients, reaching a diagnosis detection rate of $30 \%$. In conclusion, genetic defects in dyshormonogenesis, mainly in DUOX2, were the main genetic cause of $\mathrm{CH}$ in the
\end{abstract}

Correspondence to: Dr Huijuan Wang, The National Engineering Research Center for Miniaturized Detection Systems, College of Life Science, Northwest University, 229 North Taibai Road, Xi'an, Shaanxi 710069, P.R. China

E-mail:whj@nwu.edu.cn

Dr Xiaoli Yan, Endocrine Department, Xi'an Children's Hospital, 69 Xijuyuan Lane, Xi'an, Shaanxi 710003, P.R. China

E-mail: yanxiaoli3097@126.com

Key words: congenital hypothyroidism, dyshormonogenesis, oligogenicity, $D U O X 2$, next-generation sequencing
Chinese population. Oligogenicity is highly involved in $\mathrm{CH}$ pathogenesis and may thus be an important factor in common phenotypic variability observed in patients with $\mathrm{CH}$.

\section{Introduction}

Congenital hypothyroidism $(\mathrm{CH})$ is the most common congenital endocrine disorder with an incidence of approximately 1/2,000-4,000 newborns (1). According to the locations of lesions, $\mathrm{CH}$ can be classified into primary, central and peripheral hypothyroidism $(1,2)$. Primary hypothyroidism accounts for $>95 \%$ of $\mathrm{CH}$ cases (3), the majority of which (80-85\%) are caused by alterations occurring during gland organogenesis. These alterations result in thyroid dysgenesis (TD) $(1,4)$. The remaining cases $(15-20 \%)$ are attributed to inborn defects in thyroid hormone synthesis. These defects are collectively known as dyshormonogenesis (DH) and are generally characterized by either goiter or normal thyroid glands $(1,5,6)$. By contrast, central and peripheral hypothyroidisms are rare disorders.

Considerable progress has been made in the understanding of $\mathrm{CH}$ pathophysiology. Although most cases of $\mathrm{CH}$ occur sporadically, approximately $20 \%$ are familial and caused by genetic abnormalities $(1,2)$. For TD, approximately $2-5 \%$ of reported cases have a genetic origin. Genes associated with TD (PAX8, NKX2-1/TTF-1, FOXE1/TTF-2, NKX2-5 and TSHR) play important roles during thyroid morphogenesis $(1,4)$. The molecular mechanism of $\mathrm{DH}$ has been well characterized and most of the cases have been linked to mutations in genes involved in thyroid hormone synthesis (TG, TPO, DUOX1/2, DUOXA2, SLC5A5, SLC26A4/PDS, IYD/DEHAL1 and $S E C I S B P 2)$. These mutations are usually transmitted in an autosomal recessive mode $(1,5,7)$. The underlying molecular basis of central and peripheral hypothyroidism remains unclear, although genetic ascertainment is possible in some cases $(1,6)$. Mutations have been reported in genes controlling the biosynthetic pathway of thyroid stimulating hormone $(\mathrm{TSH} ; \mathrm{TSHB}$, TRHR and IGSF1), pituitary development (POU1F1, PROP1, HESX1, LHX3, LHX4 and SOX3) $(1,6)$ and thyroid hormone transport or action (SLC16A2/MCT8, THRB and THRA) (1). Other genes (FOXI1, GLIS3, UBRI and ZNF252P) have been 
reported in cases with syndromic hypothyroidism or transient $\mathrm{CH}$ and may be involved in $\mathrm{CH}$ (8-13). These causative genes and their functions are described in Table SI.

Although $\mathrm{CH}$ can be classified as a disease with a strong genetic component, many issues remain unresolved. One is the commonly observed variable phenotype-genotype correlations in patients $(5,14)$. This phenotypic or genetic heterogeneity suggests that mono- and polygenic factors and environmental modulators have roles in the determination of disease severity $(4,5)$. Some cases have oligogenic mutations apart from single-gene mutations and demonstrate heterogeneous phenotypes to those carrying monogenic mutations (15-17). These cases may not be inherited in a monogenic manner; that is, a digenic or oligogenic inheritance may be considered, or mutations may occur, acting as a genetic modifiers $(18,19)$. However, no definite evidence is able to prove this phenomenon. Next-generation sequencing (NGS) can be used for the simultaneous sequencing of multiple genes in a single sample and is useful in determining mutations in multiple genes that are potentially associated with diseases $(20,21)$. Thus, NGS is a powerful tool for unraveling the pathogeneses of complex diseases. Given the genetic complexity and heterogeneity of $\mathrm{CH}$, all known causative genes should be comprehensively screened for mutations for the proper understanding of $\mathrm{CH}$ pathogenesis.

According to the largest national newborn screening program between 2013 and 2015, the total incidence rate of $\mathrm{CH}$ in China is 4.13 per 10,000 live births, which is higher than the worldwide level $(22,23)$. To date, the comprehensive screening of the known pathogenic genes in Chinese patients is limited. The present study designed a targeted NGS panel including $29 \mathrm{CH}$-related genes to screen mutations in a Chinese patient cohort from Shaanxi Province, China.

\section{Materials and methods}

Subjects. A total of 43 patients with $\mathrm{CH}$ from 42 families were recruited in Xi'an Children's Hospital and Chang'an Hospital, Xi'an, China, between October 2015 and August 2016. The age of the patients at the time of the study was 3 months-13 years. The inclusion criteria were: Positive neonatal screening with a diagnosis of $\mathrm{CH}$ confirmed by serum thyroid function tests at 2-4 weeks of age. Neonatal screening for $\mathrm{CH}$ was taken from $72 \mathrm{~h}$ to 7 days after birth. Blood samples were collected from the heel to determine TSH levels by using time-resolved fluorescence assay (PerkinElmer, Inc.). Newborns (2-4 weeks) with TSH levels of $>10 \mu \mathrm{IU} / \mathrm{ml}$ were recalled for the re-examination of serum TSH and FT4 levels by electrochemiluminescence assay (Cobas 6000, Roche Diagnostics). $\mathrm{CH}$ diagnosis was based on elevated serum TSH ( $>7.5 \mu \mathrm{IU} / \mathrm{ml}$; normal: $0.27-4.20 \mu \mathrm{IU} / \mathrm{ml}$ ) and decreased FT4 levels (0.5-7.1 pmol/1; normal: 12-22 pmol/l). Levothyroxine (L-T4) treatment was initiated when elevated TSH level (>10 $\mu \mathrm{IU} / \mathrm{ml}$ ) was confirmed. All the patients had a phenotypical classification by thyroid ultrasonography performed during the neonatal period prior to treatment. Additional information on the possible existence of thyroid disease in family members was collected in all cases. The neonatal screening, diagnosis and follow-up of each patient were conducted in the same hospital: Xi'an Children's or Chang'an
Hospital. In addition, 100 subjects with normal FT4 and TSH levels and undergoing neonate thyroid screening were included in the normal control group. They were all Han Chinese from Shaanxi, China and consisted of 45 males and 55 females with a mean age of 5 days (4-10 days) on the day of sample collection for neonatal screening. Blood samples from the fathers and mothers of 20 patients were collected for segregation analysis. At the time of the study, the mean age of these fathers and mothers was 30.7 years (22-42 years) and 29.3 years (20-45 years), respectively. The parents of all the participants gave written informed consent in accordance with the Declaration of Helsinki. The study was approved by the Medical Ethics Committees of Xi'an Children's Hospital and Chang'an Hospital.

DNA extraction and sequencing. Blood samples were collected from recruited patients, their family members and the control subjects and stored in EDTA tubes. Genomic DNA was extracted and analyzed as previously described (24).

According to the previous findings described in published literature (1,4-6) and the retrieval results of the Human Gene Mutation Database (HGMD Professional 2016, http://www. hgmd.cf.ac.uk/ac/index.php), 29 causative genes (PAX8, FOXE1, NKX2-5, TSHR, NKX2-1, DUOX2, DUOXA2, TPO, SLC26A4, FOXI1, TG, SLC5A5, IYD, SECISBP 2, TSHB, IGSF1, TRHR, HESX1, LHX3, LHX4, POU1F1, PROP1, SOX3, THRB, THRA, SLC16A2, GLIS3, UBR1 and ZNF252P) associated with $\mathrm{CH}$ were selected. The recruited patients were genetically screened with a customized AmpliSeq panel (Thermo Fisher Scientific, Inc.) that included $29 \mathrm{CH}$-associated genes. The primers for the customized panel were designed with Ion AmpliSeq Designer (https://www.ampliseq. com/browse.action) for the inclusion of coding exons and the 20 flanking base pairs of the splice junctions surrounding the exons of the targeted genes. A total of 457 amplified amplicons were obtained at each sequencing run. Amplicon length was 125-374 bp (median 358 bp; Table SII). The amplicon library preparation and DNA template preparation and enrichment were conducted according to the manufacturers' protocols. DNA sequencing was performed with an Ion Torrent PGM instrument. An Ion PGM 200 sequencing kit and Ion $316^{\mathrm{TM}}$ Chip (Thermo Fisher Scientific, Inc.) were used, according to the manufacturers' protocols.

Variant detection and prioritization. Raw data were processed by the Torrent Suite software (version 5.0.4; Thermo Fisher Scientific, Inc.) for the generation of sequence reads. Each read was aligned to the hg19 human reference genome for the detection of variants. Called variants were functionally annotated with Ion Reporter (https://ionreporter.lifetechnologies.com/ir/secure/home.html) and ANNOVAR package (http://wannovar.wglab.org/). Identified variants were filtered as follows: i) Synonymous variants and nonsplice variants in the intronic region were excluded; ii) variants with minor allele frequencies (MAF) of $\leq 0.01$ or no MAF values in the dbSNP database (http://www.ncbi.nlm.nih.gov/projects/SNP/), 1000 Genomes Project (http://ftp.ncbi.nih.gov/), Exome Sequencing Project (http://evs.gs.washington.edu/EVS/) and the Genome Aggregation Database (gnomAD, http://gnomad.broadinstitute.org/) were selected; iii) variants without rs numbers 
in the dbSNP database were considered novel rare variants; iv) $\mathrm{CH}$-associated variants reported in the published literature or by the HGMD database (HGMD Professional 2019.3) were selected even if ii) or iv) was not met; v) all selected variants were validated through Sanger sequencing with ABI3500 xL Dx (Applied Biosystems, Thermo Fisher Scientific, Inc.); and vi) all validated novel variants were determined in the normal control by Sanger sequencing and were filtered by MAF $\leq 0.01$. The present study was conducted on the basis of these prioritization criteria. The prioritized variants were detected in the parental samples of patients for the verification of the inheritance of variants and segregation with phenotype.

Bioinformatics and statistical analysis. The possible functional effects of detected variants were assessed by in silico programs. For missense or indel variants, five in silico tools were used, including Polymorphism Phenotyping v2 (http://genetics.bwh. harvard.edu/pph2/ index.shtml), MutationTaster (http://www. mutationtaster.org/), Rare Exome Variant Ensemble Learner (https://sites.google.com/site/revelgenomics/) and Mendelian Clinically Applicable Pathogenicity (http://bejerano.stanford. edu/MCAP/). For the splicing variants, the deleterious effect on RNA splicing was predicted with MaxEntScan (http://genes.mit. edu/burgelab/maxent/Xmaxentscan_scoreseq.html), Berkeley Drosophila Genome Project (http://www.fruitfly.org/seq_tools/ splice.html) and NetGene2 (http://www.cbs.dtu.dk/services/ NetGene2/).

The evolutionary conservation analysis was performed using the CLC Sequence Viewer 6.5.2 software (CLC bio, Qiagen AB). Protein domains and structures were obtained from UniProt and InterPor Knowledgebase (http://www. uniprot. orssswq333 g/; http://www.ebi.ac.uk/interpro/).

The pathogenicity of each variant was assessed according to the standards described by the American College of Medical Genetics (ACMG) (25).

The clinical features of the two groups (such as patients with DUOX2 mutation and patients without DUOX2 mutation) were compared through a Mann-Whitney test for nonparametric values. $\mathrm{P}<0.05$ was considered to indicate a statistically significant difference.

\section{Results}

Demographic and clinical characteristics of the patients. The present study included 43 Chinese Han patients diagnosed with primary $\mathrm{CH}$. The recorded demographic data and clinical features of the patients are presented in Table I: The enrolled patients consisted of 25 females and 18 males, aged 3 months to 13 years. All of the cases were from unrelated families with no histories of thyroid diseases, apart from patients 32 and 33, who were monozygotic twins. Thyroid ultrasonography suggested that 11 patients had TD, of which 6 had athyreosis, 4 had hypoplasia and 1 had ectopy. A total of 32 cases had eutopically located glands in situ (GIS), 18 of which had normal thyroid sizes and 14 had goiter (Table I).

Sequencing data analysis. NGS analysis was performed on the 43 patients with $\mathrm{CH}$ and the result showed that the number of mapped reads for individual samples was 149,698-1,458,751 (median: 394571, $n=43$ ). The percentages of the on-target sequences in each sample all had a median of $99 \%$, with an average base coverage depth ranging from 215.9-2913x in individual samples. The average total coverages of all the targeted bases were $94.80 \%$ at $20 \mathrm{x}, 87.06 \%$ at $100 \mathrm{x}$ and $57.51 \%$ at $500 x$. Coverage was also uniform across all samples. On average, $94.8 \%$ of the called bases had a quality score of $\geq$ Q20 (Table SIII).

In 25 genes $(86.2 \%)$, all of the targeted amplicons were covered by 20 reads or more (Fig. S1). Exons that were covered less than 20x or missed by the NGS were subjected to Sanger sequencing (Table SIV).

Variant detection and mutation spectrum. Following functional filtering, 47 rare nonpolymorphic variants were identified in 31 of 43 patients $(71 \%)$. These variants were distributed in 9 genes, including $6 \mathrm{DH}$-associated genes (DUOX2, TG, TPO, SLC26A4, DUOXA2 and SLC5A5), 1 gene associated with TD $(T S H R)$ and 2 genes associated with central hypothyroidism (PROP1 and TRHR, Fig. 1A). Notably, all variants were detected in heterozygous status in patients. Various types of mutations have been detected and most of them were missense variants (Table II; Fig. 1A). A total of 8 novel variants were identified (Table II; Fig. S2); these were absent in the local control samples. The 39 remaining variants had been reported in HGMD, dbSNP, gnomAD and/or 1000 human genome databases.

Of the 9 mutated genes, the gene with the highest number of variants was DUOX2, followed by $T G, T P O$ and $T S H R$ (Fig. 1A). Twenty variants in DUOX2 were identified in 19 cases $(19 / 43,44 \%)$, that is, 17 patients with GIS and 2 patients with TD (Fig. 1B). Of these patients, 10 (10/43, 23\%) carried $\geq 2$ different heterozygous variants in $D U O X 2$. A total of 6 patients carried $D U O X 2$ mutation(s) in association with mutation(s) in $T G(\mathrm{n}=4)$ or SLC26A4 $(\mathrm{n}=2)$. The most common mutation was p.R1110Q (DUOX2: c.3329G $>$ A), which was found in 5 patients, accounting for $11 \%$ of all the cases. Of the 3 novel variants in DUOX2, p.T803fs was a frameshift mutation and had a potential deleterious effect on protein function and p.D137E and p.E389K were missense mutations located in the peroxidase-like domain (Fig. S3A).

A total of 9 variants in $T G$ were identified in $8 \mathrm{CH}$ patients $(8 / 43,18.6 \%), 2$ of which had $\geq 2 T G$ variants. Apart from carrying $T G$ mutation(s), 6 cases also had mutation(s) in genes associated with DH (SLC26A4, DUOX2,DUOXA2 and TPO).

A total of 6 TPO variants were separately found in 6 patients $(6 / 43,14 \%)$ in heterozygous status. All but 1 patient had a TPO mutation in association with mutation(s) in different genes. A total of 2 novel variants, p.S309P and p.S571R, were located in a myeloperoxidase-like domain, the catalytic site of the enzyme (Fig. S3B).

A total of $4 T S H R$ variants were found in 2 patients and were compound heterozygotes for 2 different TSHR mutations. The TSHR variant p.R450H was a recurrent inactivating mutation (26) and p.C176R and p.K618 were novel. p.C176R is located in the leucine-rich repeat region of the extracellular domain and responsible for high-affinity hormone binding and p.R528S and p.K618* are located in the cytoplasmic loops (Fig. S3C).

Patients with GIS had a higher tendency to be affected with mutations than patients with TD [25/32 (78\%) vs. 6/11 


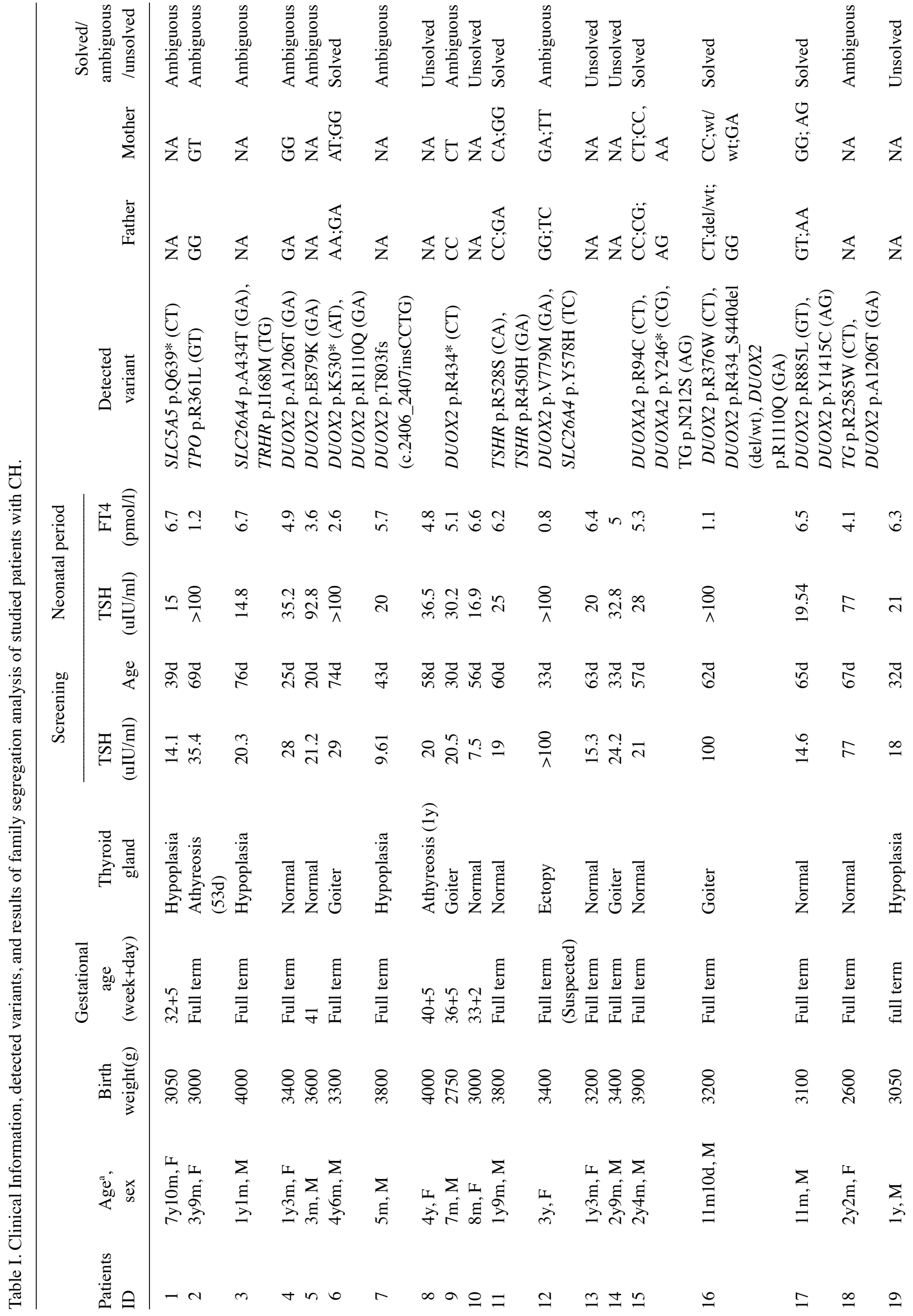




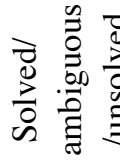

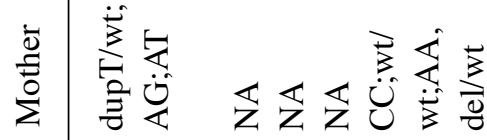

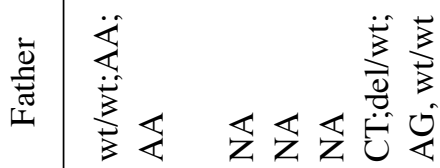

0

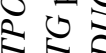

总

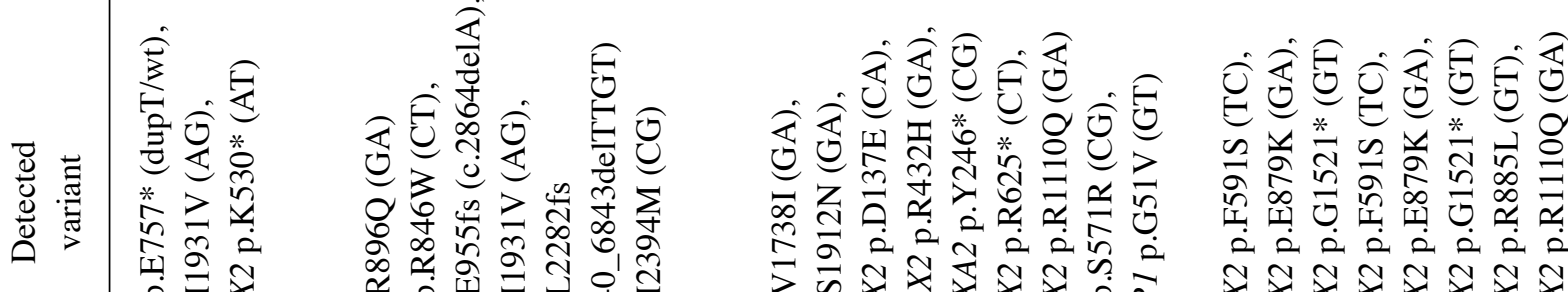

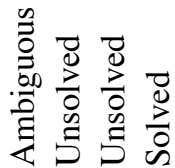

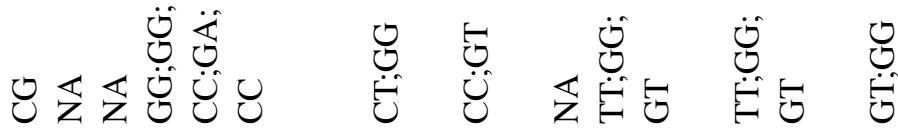

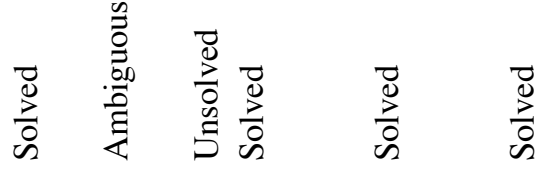

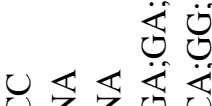

$\underset{ن}{\circlearrowright}$

ত্ঠ்

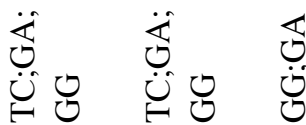

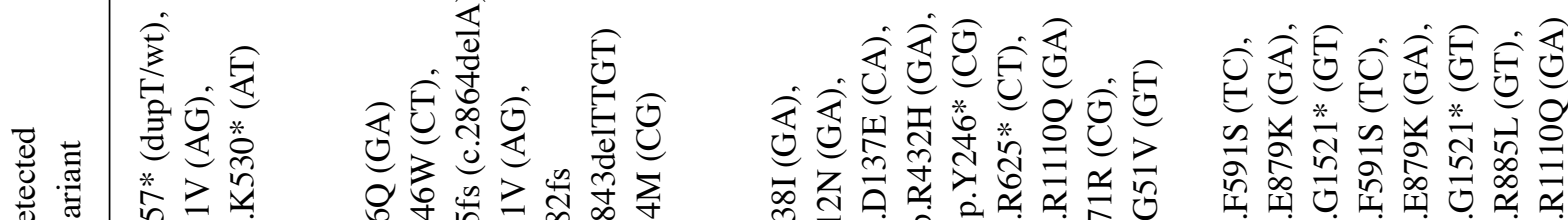

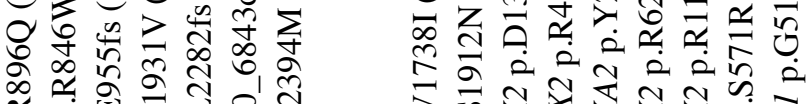

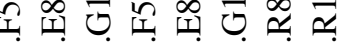

$2 \dot{2} \dot{2} \dot{2} \dot{2}$

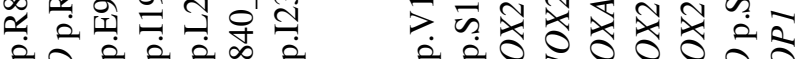

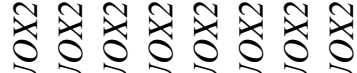

Uैं

จุด

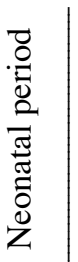

庄营竞

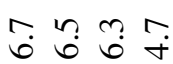

$\frac{8}{\wedge} \frac{8}{\wedge} m \frac{n}{8}$

$\stackrel{8}{\wedge}$

$\stackrel{m}{\infty}$

$\stackrel{n}{\stackrel{n}{n}}$

离

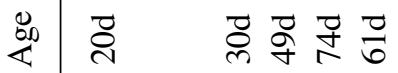

तై

$\begin{array}{lll}\vec{\infty} & \vec{\infty} & \vec{n} \\ i & \mathbb{n}\end{array}$

$\sqrt[7]{6} \quad \frac{7}{7}$

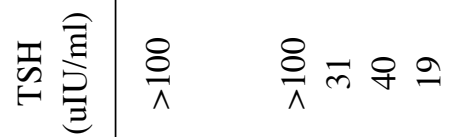

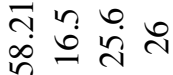

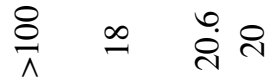

กับ

ตำ

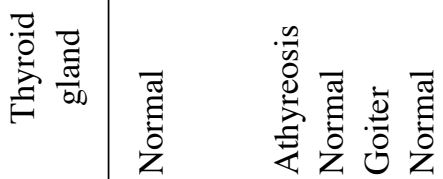

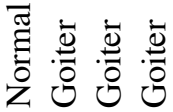

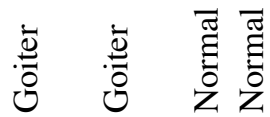

हี

:

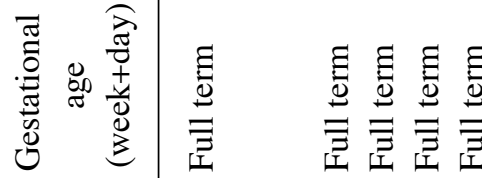

芩

言竎志。

Е

氙苟

\&

ঠ্ল

\&্

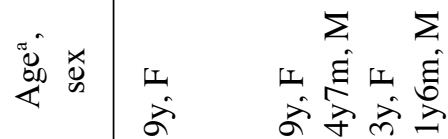

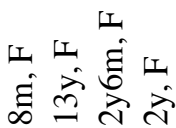

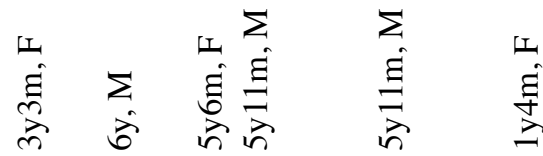

菢

สิ

ํํํํำ

ते ำ

$m$

mे 


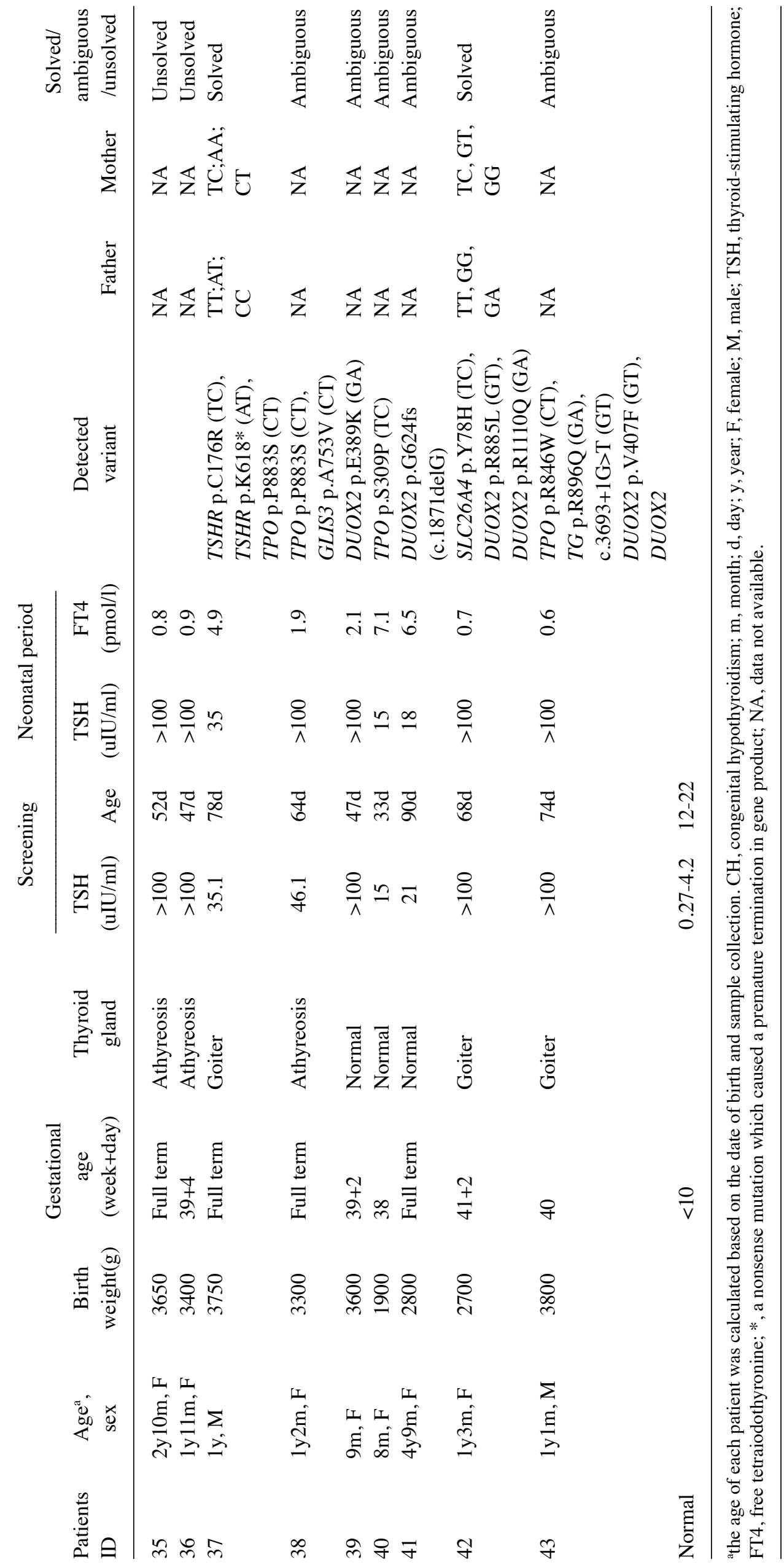


A

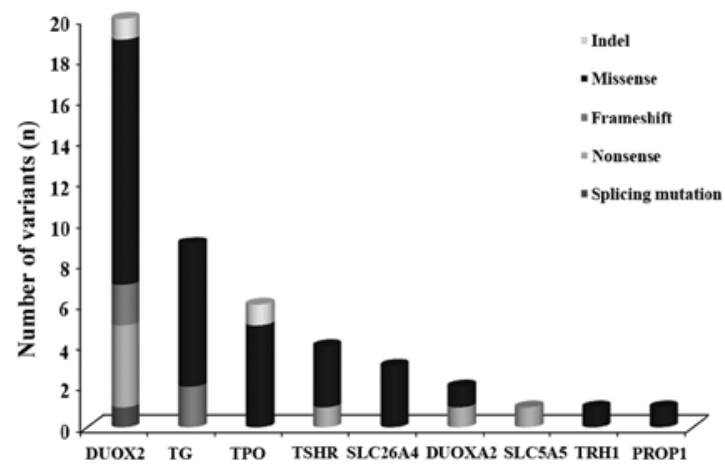

B

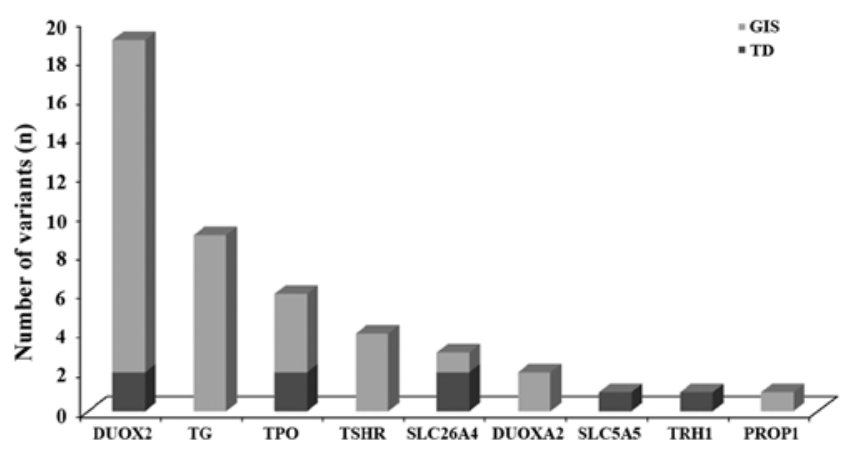

Figure 1. (A) Number of variants identified in each candidate gene according to the mutation type. (B) Number of variants identified in each candidate gene according to the $\mathrm{CH}$ phenotype. $\mathrm{CH}$, congenital hypothyroidism; GIS, $\mathrm{CH}$ with gland in situ; TD, $\mathrm{CH}$ with thyroid dysgenesis.

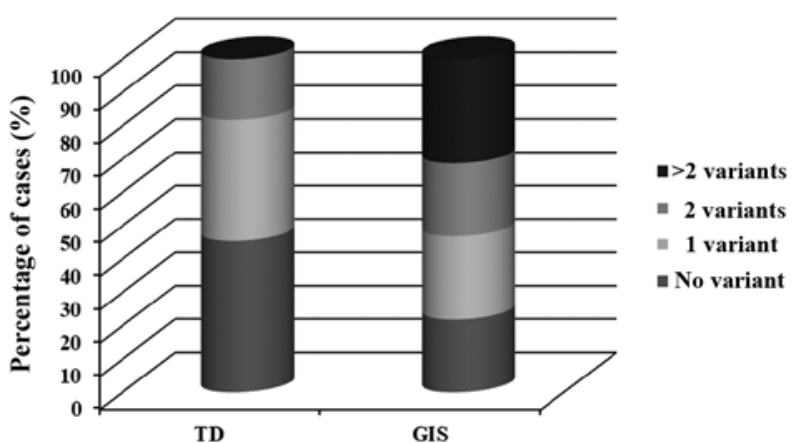

Figure 2. Percent distribution of detected variants according to the type of $\mathrm{CH}$. $\mathrm{CH}$, congenital hypothyroidism; GIS, $\mathrm{CH}$ with gland in situ; TD, $\mathrm{CH}$ with thyroid dysgenesis.

(54\%), Fig. 2]. Variants in TG, TSHR, DUOXA2, SLC5A5 and $P R O P 1$ genes were found exclusively in patients with GIS, and 1 variant in TRHR was found in patients with TD. Other genes, including DUOX2, TPO and SLC26A4, were associated with either dysgenesis or GIS phenotype (Table II and Fig. 1B). The variants detected in the 6 patients with TD (two athyreosis, two hypoplasia and one ectopy) were all located in genes associated with DH. A total of 12 patients $(12 / 43,28 \%)$ carried only 1 heterozygous variant and 19 cases $(19 / 43,44 \%)$ had $\geq 2$ variants, 8 of which were monogenic (having mutations in the same gene) and 11 were oligogenic (having mutations in different genes, Table III).

Pathogenicity assessment. The pathogenicity of the detected variants was classified in accordance with ACMG standards and guidelines (Table II, Tables SV and SVI). Among the 47 variants, 25 were classified as pathogenic (P) or likely pathogenic (LP), namely, 16 in DUOX2, 4 in TSHR, 2 in TPO, 2 in $T G$ and 1 in DUOXA2 gene. A total of 21 variants were classified as variants of uncertain significance (VUS) and 1 variant in TPO was classified as likely benign. Among the 8 novel variants, 4 were classified as P (p.C176R and p.K618* in $T S H R$, p.T803fs in DUOX2) or LP (p.D137E in DUOX2), the other were classified as VUS.

Genotype and phenotype relationship. Through family segregation and pathogenicity assessment, 13 cases (patients 6,11 ,
15,17, 16, 28, 29, 32, 33, 34, 37 and 42) were considered 'solved,' reaching a diagnosis detection rate of $30 \%$ (Tables I and III, and Fig. S1). These 'solved' cases all carried at least 2 pathogenic variants in the same gene, which were of either paternal or maternal origin, but not from a single parent, following the identification of a decisive link between genotype and phenotype. A total of 18 cases (41.9\%) were considered 'ambiguous' owing to the weak link between genotype and phenotype. In addition, 12 cases were considered 'unsolved' because they carried no mutations in any of the listed genes.

Among the solved cases, 8 were monogenic and 5 were oligogenic. The main pathogenic genes were DUOX2 $(\mathrm{n}=9)$, TSHR $(\mathrm{n}=2)$, DUOXA2 $(\mathrm{n}=1)$ and TG $(\mathrm{n}=1)$. Notably, all of the 'solved' cases were patients with GIS (Tables I and III). Therefore, the diagnosis rate in patients with GIS patients was $40.6 \%$ (13/32; Table III).

According to the number of variants carried, the studied cases were classified into different groups and the serum levels of TSH and FT4 were compared among these groups (Fig. SIV). The results showed that only the average serum TSH level of patients with TG mutation at diagnosis were significantly higher than those without $T G$ mutation $(49.54 \mu \mathrm{IU} / \mathrm{ml}$ vs. $68.71 \mu \mathrm{IU} / \mathrm{ml}, \mathrm{P}=0.037$, Fig. S4A-b). The average serum levels of FT4 of patients with monogenic mutation at diagnosis were higher compared with patients with oligogenic mutations, but the difference was not statistically significant $(\mathrm{P}=0.05$, Fig. S4D-c).

\section{Discussion}

To the best of our knowledge, the present study is the first in which the currently largest targeted NGS panel containing 29 known causative genes was used for the comprehensive examination of the mutation spectrum of Han Chinese $\mathrm{CH}$ patients. The present study found a high mutation rate $(44 \%)$ in primary $\mathrm{CH}$ patients and most of the mutations $(91.5 \%)$ were identified in genes associated with $\mathrm{DH}$. In addition, mutations in genes associated with thyroid development or transcription were rarely identified. The majority of $\mathrm{CH}$ were caused by TD and $<20 \%$ of cases showed strong genetic predisposition $(1,2)$. However, in the patient cohort, $\mathrm{DH}(\mathrm{n}=32)$ was more common than TD $(n=11)$. This result is in agreement with the data reported in China (27-31). Given that DH is largely caused by genetic 


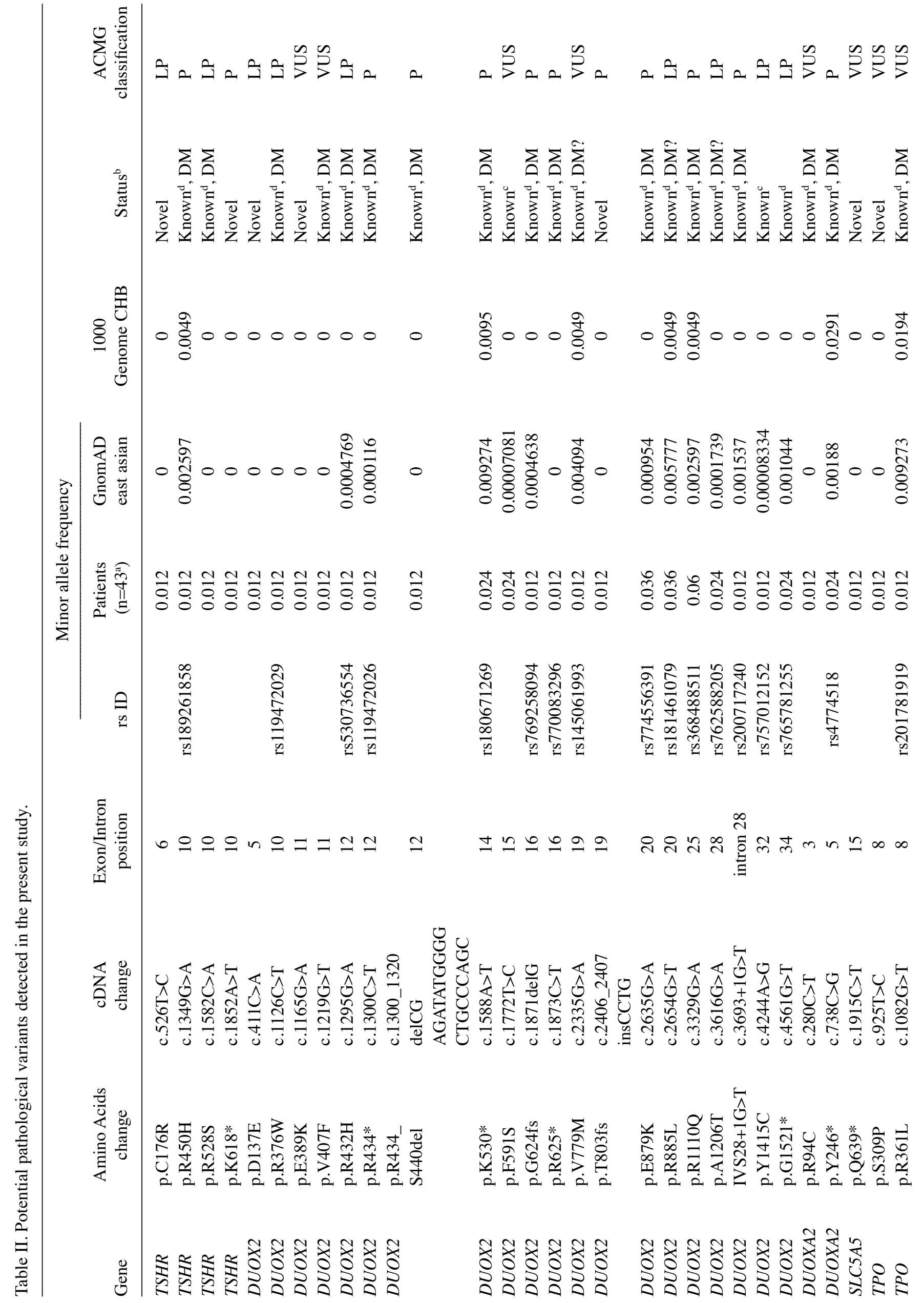




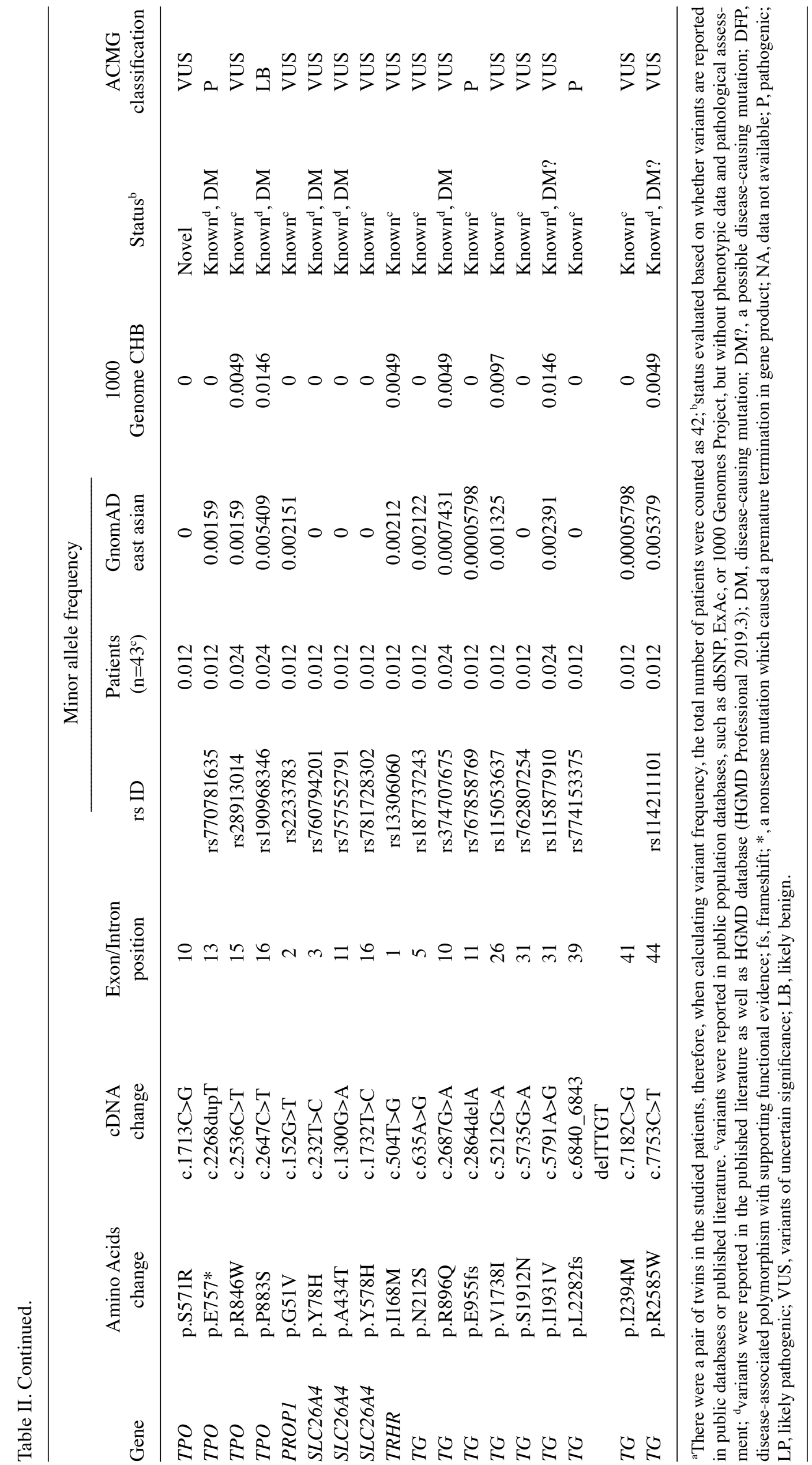


Table III. Mutation spectrum of 'solved' and 'ambiguous' cases with $\mathrm{CH}$.

Solved ( $\mathrm{n}=13)$, all were $\mathrm{CH}$ with GIS

\begin{tabular}{|c|c|c|c|c|c|c|c|c|c|}
\hline \multicolumn{3}{|c|}{ Monogenic $(\mathrm{n}=8)$} & \multicolumn{7}{|c|}{ Oligogenic $(n=5)$} \\
\hline Gene & $\begin{array}{c}\text { Number of } \\
\text { variants }\end{array}$ & $\begin{array}{c}\text { Number of } \\
\text { patients }\end{array}$ & Gene1 & $\begin{array}{l}\text { Number of } \\
\text { variants }\end{array}$ & Gene2 & $\begin{array}{l}\text { Number of } \\
\text { variants }\end{array}$ & Gene3 & $\begin{array}{c}\text { Number of } \\
\text { variants }\end{array}$ & $\begin{array}{c}\text { Number of } \\
\text { patients }\end{array}$ \\
\hline TSHR & 2 & 1 & TSHR & 2 & $T P O$ & 1 & & & 1 \\
\hline DUOX2 & 2 & 4 & DUOXA2 & 2 & $T G$ & 1 & & & 1 \\
\hline \multirow[t]{3}{*}{ DUOX2 } & 3 & 3 & DUOX2 & 2 & SLC26A4 & 1 & & & 1 \\
\hline & & & DUOX2 & 2 & $T G$ & 2 & DUOXA2 & 1 & 1 \\
\hline & & & $T G$ & 3 & $T P O$ & 1 & & & 1 \\
\hline
\end{tabular}

Ambiguous $(\mathrm{n}=18)$

\begin{tabular}{|c|c|c|c|c|c|c|c|c|c|}
\hline \multicolumn{3}{|c|}{ Monogenic(n=12) } & \multicolumn{7}{|c|}{ Oligogenic $(n=6)$} \\
\hline Gene & $\begin{array}{c}\text { Number of } \\
\text { variants }\end{array}$ & $\begin{array}{c}\text { Number of } \\
\text { patients }\end{array}$ & Gene1 & $\begin{array}{l}\text { Number of } \\
\text { variants }\end{array}$ & Gene2 & $\begin{array}{c}\text { Number of } \\
\text { variants }\end{array}$ & Gene3 & $\begin{array}{c}\text { Number of } \\
\text { variants }\end{array}$ & $\begin{array}{c}\text { Number of } \\
\text { patients }\end{array}$ \\
\hline DUOX2 & 1 & 6 & SLC26A4 & 1 & TRHI & 1 & & & 1 \\
\hline$T G$ & 1 & 2 & DUOX2 & 1 & SLC26A4 & 1 & & & 1 \\
\hline TPO & 1 & 3 & DUOX2 & 1 & $T G$ & 1 & & & 1 \\
\hline \multirow[t]{3}{*}{ SLC5A5 } & 1 & 1 & DUOX2 & 1 & $T G$ & 1 & $T P O$ & 1 & 1 \\
\hline & & & DUOX2 & 2 & $T G$ & 1 & TPO & 1 & 1 \\
\hline & & & ТPO & 1 & $P R O P 1$ & 1 & & & 1 \\
\hline
\end{tabular}

$\mathrm{CH}$, congenital hypothyroidism.

defects and considered a hereditary disease, a majority of $\mathrm{CH}$ cases in Chinese are hereditary and have a strong genetic origin. In the present study, mutation detection rate in $\mathrm{CH}$ patients with DH was $78 \%$ and the diagnosis rate in DH was $40.6 \%$. Notably, the 6 patients with TD harbored mutations that were all associated with DH, 2 of whom had athyreosis. Patients with TD, especially those with athyreosis, are unlikely to carry variants associated with DH. However, in the present study, patients were subjected to thyroid ultrasonography rather than to whole-body nuclear magnetic resonance scanning for the examination of thyroid morphology. Thus, those examined as athyreosis could not be excluded for the likelihood of ectopy. Similarly, other studies have found variants associated with $\mathrm{DH}$ in patients with athyreosis $(30,32)$. In addition, 2 individual variants in genes associated with pituitary development or central $\mathrm{CH}$ (PROPI and TRHR) respectively were found in $1 \mathrm{CH}$ patient with GIS and 1 patient with TD, and both variants co-occurred with genetic variants associated with $\mathrm{DH}$. Currently, it is difficult to be sure that the genetic defects associated with $\mathrm{DH}$ contribute to the development of TD or the potential effect of variants associated with pituitary development on primary $\mathrm{CH}$. However, the findings of the present study validated the complicated pathological mechanism of $\mathrm{CH}$. Thus, studies on the genetic origin of TD or DH diseases should not be limited to well-known causative genes $(4,33)$.

In the present study, DUOX 2 was the most common genetic alteration identified in $\mathrm{CH}$ patients. The detection rate of DUOX2 mutation in the studied $\mathrm{CH}$ cohort and the GIS patients were $44 \%(19 / 43)$ and $53.1 \%$ (17/32), respectively, most of which carried $\geq 2$ DUOX 2 mutations. This finding was in accordance with those previously reported showing that DUOX2 mutation is the leading genetic cause of $\mathrm{CH}$ in Asian populations, including other Han Chinese, Japanese and Koreans; the detection rate of DUOX2 in patients from Asian populations is $16.5-60 \%$ and $\leq 83 \%$ in patients with DH (27,29,31,34-40). TG and TPO mutations were also commonly found in the studied cohort. However, a majority of these mutations were either separately presented at a heterozygous status or detected with mutations in different genes. Thus, TG and TPO may act as contributing genetic factors apart from being the main genetic causes of $\mathrm{CH}$ in the studied cohort. In some Caucasian cohorts, TPO has been identified as the main genetic cause of $\mathrm{CH}$ (41-43). In addition, loss-of-function (LOF) mutations in the TSH receptor (TSHR) gene were identified as the most frequent cause of TSH resistance, leading to a wide spectrum of phenotypes ranging from severe $\mathrm{CH}$ to mild euthyroid hyperthyrotropinemia $(26,44)$. In the present study, 2 patients with $\mathrm{CH}$ who were compound heterozygotes for 2 different TSHR mutations ( 1 for p.R528S and p.R450H and 1 for p.C176R and p.K618*) demonstrated mild clinical phenotypes $(5 \mathrm{pmol} / \mathrm{l} \leq \mathrm{FT} 4<10 \mathrm{pmol} / \mathrm{l})(45)$. This finding is in agreement with previous studies reporting that compound heterozygotes of less severe LOF mutations are usually associated with 
mild/borderline forms of hypothyroidism, wherein an appropriate increase in TSH serum levels can compensate for the reduced sensitivity of the thyroid (partially or fully compensated TSH resistance) $(26,44,46,47)$. Previously reported clinical cases with TSHR mutations are always characterized by normal-sized or hypoplastic thyroid gland $(26,46,47)$. However, in the present study, patient 37 suffered from goiter. In addition to TSHR mutations, this patient also carried a heterozygous variant in $T P O$, which may be the reason leading to this phenotypic variability.

At present, patients with $\mathrm{CH}$ caused by genetic defects are considered to be inherited in a monogenic manner. However, phenotypic variability observed in patients with same mutations indicated the influence of other factors, such as genetic heterogeneity $(15,34,42,43,48-50)$. The present study found a high percentage $(25.6 \%)$ of involvement of oligogenic mutations in studied cases, which is similar to that of previous studies simultaneously assessing multiple genes (15,30-32). These studies reported frequent oligogenic involvement in $\mathrm{CH}$, with oligogenic mutations in $20-43.5 \%$ of patients with $\mathrm{CH}$ and GIS and/or patients with TD in different ethnic populations. In addition, among the 13 'solved' cases through family segregation and pathogenicity assessment, 5 cases carried oligogenic mutations and none of the mutations was inherited from a single parent. These findings suggested that, not only monogenic inheritance, but also digenic or oligogenic inheritance is involved in the pathogenesis of $\mathrm{CH}$. However, available evidence is insufficient for oligogenic inheritance verification, and protein-protein interaction for the two proteins or genes, pedigree data, animal models or very specific functional experiments are key factors (18). At present, only 1 study performed in mice demonstrated a multigenic origin of $\mathrm{CH}$ with TD (51). Therefore, further studies are required for the validation of the role and mechanism of oligogenicity in $\mathrm{CH}$ pathogenesis.

The data of the present study were compared with those of several similar studies that analyzed the mutation spectrum of $\mathrm{CH}$ patients in China by using NGS (29-31). The investigated patients in the present study were from northwestern China (Shaanxi Province), whereas those in the previous studies were mainly from southern China (Jiangsu and Guangxi Provinces). The general mutation profiles of patients with $\mathrm{CH}$ demonstrated by these studies were similar. For example, the total mutation rate in $\mathrm{CH}$ patients reported in these studies was relatively high, i.e., $48.5 \%$ (29), $65.09 \%$ (31), $80.9 \%$ (30) and $72 \%$ (the present study). DUOX 2 mutations were the prevalent genetic alterations in these studies, with a mutation rate of $31.8 \%$ (29), 31.3\% (31), $60 \%$ (30) and 44\% (the present study). Therefore, the region (or population)-specific characteristics in patients with $\mathrm{CH}$ from these studies could not be ascertained. However, some different findings in the present study were observed. For example, DUOX 2 and TG mutations were the first and second most common mutations detected in all these studies. However, the third and fourth most common genetic mutations were different. In the present study, the third and fourth most common genetic mutation were TPO and TSHR mutations, respectively. in addition, the third and fourth most common genetic mutations reported by Long et al (31) were TSHR and GNAS mutations, respectively and those by Sun et al (30) were TPO and DUOXAl mutations, respectively.
These discrepancies may be caused by the relatively small sample size, sampling criteria and/or different targeted genes determined.

Several limitations were observed in the present study. The sample size was relatively small, and most patients were too young to exhibit clinical phenotypes. Thus, determining the clinical significance of the detected mutations was difficult. Pedigree analysis was not performed in all the cases carrying mutations and evidence to support the pathogenicity of detected variants was insufficient. The diagnosis detection rate in the present study would be $>30 \%$ were these requirements met. Finally, in vitro functional study of novel variants identified in the current study should be carried out.

In conclusion, using the currently largest targeted NGS panel containing 29 known genes, the mutation spectrum of 43 Han Chinese patients with $\mathrm{CH}$ was comprehensively determined. The main findings showed that DH other than TD is the common cause of $\mathrm{CH}$ in Chinese populations and genetic alterations associated with thyroid hormone biosynthesis, especially DUOX2 mutations, are the main genetic causes of $\mathrm{CH}$. In addition, a high percentage of involvement of oligogenic mutation in the studied cases confirmed the potential role of oligogenicity or non-Mendelian inheritance in $\mathrm{CH}$ pathogenesis.

\section{Acknowledgements}

Not applicable.

\section{Funding}

This study was supported by the National Natural Science Foundation of China (grant no. 81702483) and Shaanxi Innovative Talents Promotion Plan (grant no. 2017KJXX-33).

\section{Availability of data and materials}

The datasets used and/or analyzed during the current study are available from the corresponding author on reasonable request.

\section{Authors' contributions}

HW and XY conceived the project. YW, LirZ performed the experiment. XK and LixZ analyzed and interpreted the data. $\mathrm{CC}$ designed the study and revised the article. $\mathrm{HW}$ reviewed the literature and wrote the article. $\mathrm{YP}, \mathrm{YZ}, \mathrm{XC}$ and $\mathrm{ZH}$ were involved in sample and medical record recruitment. All authors read and approved the final manuscript.

\section{Ethics approval and consent to participate}

The parents of all participants gave written informed consent in accordance with the Declaration of Helsinki. The study was approved by the Medical Ethics Committees of Xi'an Children's Hospital and Chang'an Hospital.

\section{Patient consent for publication}

Written informed consent was obtained from the parents of all the participants for the publication of their data. 


\section{Competing interests}

The authors declare that they have no competing interests.

\section{References}

1. Rastogi MV and Lafranchi SH: Congenital hypothyroidism. Orphanet J Rare Dis 5: 17, 2010.

2. Wassner AJ and Brown RS: Congenital hypothyroidism: Recent advances. Curr Opin Endocrinol Diabetes Obes 22: 407-412, 2015

3. Nygaard B: Hyperthyroidism (primary). Bmj Clin Evid 2010 $0611,2010$.

4. Nettore IC, Cacace V, De Fusco C, Colao A and Macchia PE: The molecular causes of thyroid dysgenesis: A systematic review. J Endocrinol Invest 36: 654-664, 2013.

5. Grasberger H and Refetoff S: Genetic causes of congenital hypothyroidism due to dyshormonogenesis. Curr Opin Pediatr 23: $421-428,2011$

6. Schoenmakers N, Alatzoglou KS, Chatterjee VK and Dattani MT: Recent advances in central congenital hypothyroidism. J Endocrinol 227: R51-R71, 2015.

7. Park SM and Chatterjee VK: Genetics of congenital hypothyroidism. J Med Genet 42: 379-389, 2005.

8. Hulander M, Kiernan AE, Blomqvist SR, Carlsson P, Samuelsson EJ, Johansson BR, Steel KP and Enerbäck S: Lack of pendrin expression leads to deafness and expansion of the endolymphatic compartment in inner ears of Foxil null mutant mice. Development 130: 2013-2025, 2003.

9. Zenker M, Mayerle J, Lerch MM, Tagariello A, Zerres K, Durie PR, Beier M, Hülskamp G, Guzman C, Rehder H, et al: Deficiency of UBR1, a ubiquitin ligase of the $\mathrm{N}$-end rule pathway, causes pancreatic dysfunction, malformations and mental retardation (Johanson-Blizzard syndrome). Nat Genet 37: 1345-1350, 2005.

10. Yang T, Vidarsson H, Rodrigo-Blomqvist S, Rosengren SS, Enerback S and Smith RJ: Transcriptional control of SLC26A4 is involved in Pendred syndrome and nonsyndromic enlargement of vestibular aqueduct (DFNB4). Am J Hum Genet 80: 1055-1063, 2007.

11. Secchi LA, Mazzeu JF, Córdoba MS, Ferrari I, Ramos HE and Neves Fde A: Transient neonatal hypothyroidism in a boy with unbalanced translocationt $(8 ; 16)$. Arq Bras Endocrinol Metabol 56: 564-569, 2012.

12. Lichti-Kaiser K, ZeRuth G and Jetten AM: Transcription factor Gli-Similar 3 (GLIS3): Implications for the development of congenictal hypothyroidism. J Endocrinol Diabetes Obes 2 : $1024,2014$.

13. Kang HS, Kumar D, Liao G, Lichti-Kaiser K, Gerrish K, Liao XH, Refetoff S, Jothi R and Jetten AM: GLIS3 is indispensable for TSH/TSHR-dependent thyroid hormone biosynthesis and follicular cell proliferation. J Clin Invest 127: 4326-4337, 2017.

14. Muzza M, Rabbiosi S, Vigone MC, Zamproni I, Cirello V, Maffini MA, Maruca K, Schoenmakers N, Beccaria L, Gallo F, et al: The clinical and molecular characterization of patients with dyshormonogenic congenital hypothyroidism reveals specific diagnostic clues for DUOX2 defects. J Clin Endocrinol Metab 99: E544-E553, 2014.

15. Nicholas AK, Serra EG, Cangul H, Alyaarubi S, Ullah I, Schoenmakers E, Deeb A, Habeb AM, Almaghamsi M, Peters C, et al: Comprehensive screening of eight known causative genes in congenital hypothyroidism with gland-in-situ. J Clin Endocrinol Metab 101: 4521-4531, 2016.

16. Yoshizawa-Ogasawara A, Abe K, Ogikubo S, Narumi S, Hasegawa $\mathrm{T}$ and Satoh M: Transient congenital hypothyroidism caused by compound heterozygous mutations affecting the NADPH-oxidase domain of DUOX2. J Pediatr Endocrinol Metab 29: 363-371, 2016.

17. Zheng X, Ma SG, Qiu YL, Guo ML and Shao XJ: A novel c. $554+5 \mathrm{C}>\mathrm{T}$ mutation in the DUOXA2 gene combined with p.R885Q mutation in the DUOX2 gene causing congenital hypothyroidism. J Clin Res Pediatr Endocrinol 8: 224-227, 2016.

18. Schäffer AA: Digenic inheritance in medical genetics. J Med Genet 50: 641-652,2013.

19. Kousi M and Katsanis N: Genetic modifiers and oligogenic inheritance. Cold Spring Harb Perspect Med 5: a017145, 2015.

20. Lu JT, Campeau PM and Lee BH: Genotype-phenotype correlation-promiscuity in the era of next-generation sequencing. N Engl J Med 371: 593-596, 2014.
21. Manolio TA, Collins FS, Cox NJ, Goldstein DB, Hindorff LA, Hunter DJ, McCarthy MI, Ramos EM, Cardon LR, Chakravarti A, et al: Finding the missing heritability of complex diseases. Nature 461: 747-753, 2009.

22. Deng K, He C, Zhu J, Liang J, Li X, Xie X, Yu P, Li N, Li Q and Wang Y: Incidence of congenital hypothyroidism in China: Data from the national newborn screening program, 2013-2015. J Pediatr Endocrinol Metab 31: 601-608, 2018.

23. Hinton CF, Harris KB, Borgfeld L, Drummond-Borg M, Eaton R, Lorey F, Therrell BL, Wallace J and Pass KA: Trends in incidence rates of congenital hypothyroidism related to select demographic factors: Data from the United States, California, Massachusetts, New York, and Texas. Pediatrics 125 (Suppl 2): S37-S47, 2010.

24. Chen X, Kong X, Zhu J, Zhang T, Li Y, Ding G and Wang H: Mutational spectrum analysis of seven genes associated with thyroid dyshormonogenesis. Int J Endocrinol 2018: 8986475, 2018.

25. Richards S, Aziz N, Bale S, Bick D, Das S, Gastier-Foster J, Grody WW, Hegde M, Lyon E, Spector E, et al: Standards and guidelines for the interpretation of sequence variants: A joint consensus recommendation of the American college of medical genetics and genomics and the association for molecular pathology. Genet Med 17: 405-424, 2015.

26. Cassio A, Nicoletti A, Rizzello A, Zazzetta E, Bal M and Baldazzi L: Current loss-of-function mutations in the thyrotropin receptor gene: When to investigate, clinical effects, and treatment. J Clin Res Pediatr Endocrinol 5 (Suppl 1): 29-39, 2013.

27. Jiang H, Wu J, Ke S, Hu Y, Fei A, Zhen Y, Yu J and Zhu K High prevalence of DUOX2 gene mutations among children with congenital hypothyroidism in central China. Eur J Med Genet 59: 526-531, 2016.

28. Albert BB, Cutfield WS, Webster D, Carll J, Derraik JG, Jefferies C, Gunn AJ and Hofman PL: Etiology of increasing incidence of congenital hypothyroidism in New Zealand from 1993-2010. J Clin Endocrinol Metab 97: 3155-3160, 2012.

29. Fan X, Fu C, Shen Y, Li C, Luo S, Li Q, Luo J, Su J, Zhang S, $\mathrm{Hu} \mathrm{X}$, et al: Next-generation sequencing analysis of twelve known causative genes in congenital hypothyroidism. Clin Chim Acta 468: 76-80, 2017.

30. Sun F, Zhang JX, Yang CY, Gao GQ, Zhu WB, Han B, Zhang LL, Wan YY, Ye XP, Ma YR, et al: The genetic characteristics of congenital hypothyroidism in China by comprehensive screening of 21 candidate genes. Eur J Endocrinol 178: 623-633, 2018

31. Long W, Lu G, Zhou W, Yang Y, Zhang B, Zhou H, Jiang L and $\mathrm{Yu}$ B: Targeted next-generation sequencing of thirteen causative genes in Chinese patients with congenital hypothyroidism. Endocr J 65: 1019-1028, 2018.

32. de Filippis T, Gelmini G, Paraboschi E, Vigone MC, Di Frenna M, Marelli F, Bonomi M, Cassio A, Larizza D, Moro M, et al: A frequent oligogenic involvement in congenital hypothyroidism. Hum Mol Genet 26: 2507-2514, 2017.

33. De Felice M and Di Lauro R: Thyroid development and its disorders: Genetics and molecular mechanisms. Endocr Rev 25: 722-746, 2004.

34. Narumi S, Muroya K, Asakura Y, Aachi M and Hasegawa T: Molecular basis of thyroid dyshormonogenesis: Genetic screening in population-based Japanese patients. J Clin Endocrinol Metab 96: E1838-E1842, 2011.

35. Park KJ, Park HK, Kim YJ, Lee KR, Park JH, Park JH, Park HD, Lee SY and Kim JW: DUOX2 mutations are frequently associated with congenital hypothyroidism in the Korean population. Ann Lab Med 36: 145-153, 2016.

36. Wang F, Lu K, Yang Z, Zhang S, Lu W, Zhang L, Liu S and Yan S: Genotypes and phenotypes of congenital goitre and hypothyroidism caused by mutations in dual oxidase 2 genes. Clin Endocrinol (Oxf) 81: 452-457, 2014

37. Fu C, Zhang S, Su J, Luo S, Zheng H, Wang J, Qin H, Chen Y, Shen Y, Hu X, et al: Mutation screening of DUOX2 in Chinese patients with congenital hypothyroidism. J Endocrinol Invest 38: 1219-1224, 2015.

38. Fu C, Luo S, Zhang S, Wang J, Zheng H, Yang Q, Xie B, Hu X, Fan X, Luo J, et al: Next-generation sequencing analysis of DUOX2 in 192 Chinese subclinical congenital hypothyroidism (SCH) and $\mathrm{CH}$ patients. Clin Chim Acta 458: 30-34, 2016

39. Matsuo K, Tanahashi Y, Mukai T, Suzuki S, Tajima T, Azuma H and Fujieda K: High prevalence of DUOX2 mutations in Japanese patients with permanent congenital hypothyroidism or transient hypothyroidism. J Pediatr Endocrinol Metab 29: 807-812, 2016.

40. Tan M, Huang Y, Jiang X, Li P, Tang C, Jia X, Chen Q, Chen W, Sheng H, Feng Y, et al: The prevalence, clinical, and molecular characteristics of congenital hypothyroidism caused by DUOX2 mutations: A population-based cohort study in Guangzhou. Horm Metab Res 48: 581-588, 2016. 
41. Cangul H, Aycan Z, Olivera-Nappa A, Saglam H, Schoenmakers NA, Boelaert K, Cetinkaya S, Tarim O, Bober E, Darendeliler F, et al: Thyroid dyshormonogenesis is mainly caused by TPO mutations in consanguineous community. Clin Endocrinol (Oxf) 79: 275-281, 2013.

42. Avbelj M, Tahirovic H, Debeljak M, Kusekova M, Toromanovic A, Krzisnik C and Battelino T: High prevalence of thyroid peroxidase gene mutations in patients with thyroid dyshormonogenesis. Eur J Endocrino 156: 511-519, 2007.

43. Löf C, Patyra K, Kuulasmaa T, Vangipurapu J, Undeutsch H, Jaeschke H, Pajunen T, Kero A, Krude H, Biebermann H, et al: Detection of novel gene variants associated with congenital hypothyroidism in a Finnish patient cohort. Thyroid 26: 1215-1224, 2016.

44. Persani L, Calebiro D, Cordella D, Weber G, Gelmini G, Libri D, de Filippis T and Bonomi M: Genetics and phenomics of hypothyroidism due to TSH resistance. Mol Cell Endocrinol 322: 72-82, 2010.

45. Léger J, Olivieri A, Donaldson M, Torresani T, Krude H, van Vliet G, Polak M, and Butler G; ESPE-PES-SLEP-J SPE-APEG-APPES-ISPAE; Congenital Hypothyroidism Consensus Conference Group: European society for paediatric endocrinology consensus guidelines on screening, diagnosis, and management of congenital hypothyroidism. Horm Res Paediatr 81: 80-103, 2014

46. Kanda K, Mizuno H, Sugiyama Y, Imamine H, Togari H and Onigata K: Clinical significance of heterozygous carriers associated with compensated hypothyroidism in $\mathrm{R} 450 \mathrm{H}$, a common inactivating mutation of the thyrotropin receptor gene in Japanese. Endocrine 30: 383-388, 2006.
47. Tsunekawa K, Onigata K, Morimura T, Kasahara T, Nishiyama S, Kamoda T, Mori M, Morikawa A and Murakami M: Identification and functional analysis of novel inactivating thyrotropin receptor mutations in patients with thyrotropin resistance. Thyroid 16: 471-479, 2006.

48. Maruo Y, Takahashi H, Soeda I, Nishikura N, Matsui K, Ota Y, Mimura Y, Mori A, Sato H and Takeuchi Y: Transient congenital hypothyroidism caused by biallelic mutations of the dual oxidase 2 gene in Japanese patients detected by a neonatal screening program. J Clin Endocrinol Metab 93: 4261-4267, 2008.

49. Jin HY, Heo SH, Kim YM, Kim GH, Choi JH, Lee BH and Yoo HW: High frequency of DUOX2 mutations in ransient or permanent congenital hypothyroidism with eutopic thyroid glands. Horm Res Paediatr 82: 252-260, 2014.

50. Hu X, Chen R, Fu C, Fan X, Wang J, Qian J, Yi S, Li C, Luo J, $\mathrm{Su} \mathrm{J}$, et al: Thyroglobulin gene mutations in Chinese patients with congenital hypothyroidism. Mol Cell Endocrinol 423: 60-66, 2016.

51. Amendola E, De Luca P, Macchia PE, Terracciano D, Rosica A, Chiappetta G, Kimura S, Mansouri A, Affuso A, Arra C, et al: A mouse model demonstrates a multigenic origin of congenital hypothyroidism. Endocrinology 146: 5038-5047, 2005.

(i) $(9)$ This work is licensed under a Creative Commons Attribution-NonCommercial-NoDerivatives 4.0 International (CC BY-NC-ND 4.0) License. 